\title{
Modelling and simulation of the fatigue usage factor of $\gamma$-TiAl alloy fabricated through Laser Additive Manufacturing (LAM)
}

\author{
SA Raji ${ }^{a, b, *}$, API Popoolaa, SL Pityanac, OM Popoolad ${ }^{d}$, NKK Arthurc, M Tlotleng ${ }^{c, e}$
}

\author{
aDepartment of Chemical, Metallurgical and Materials Engineering, Tshwane University of Technology, Staatsartillerie Road, Pretoria West, \\ Pretoria, South Africa \\ ${ }^{b}$ Department of Metallurgical Engineering, Yaba College of Technology, P.M.B. 2011 Yaba, Lagos, Nigeria \\ 'National Laser Centre, Council for Scientific and Industrial Research, Meiring Naudé Road, Brummeria, Pretoria 0184, South Africa \\ ${ }^{d}$ Department of Electrical Engineering, Centre for Energy and Electric Power (CEEP), Tshwane University of Technology, Staatsartillerie Road, \\ Pretoria West, Pretoria, South Africa \\ eDepartment of Mechanical Engineering Science, University of Johannesburg, Auckland Park Campus, Johannesburg, South Africa \\ Email: RajiSA@tut.ac.za; rajisadiqa@gmail.com
}

\begin{abstract}
Recently, laser additive manufacturing (LAM) technologies are increasingly being applied for producing components with excellent physical and mechanical properties in the aerospace, automotive and energy industries. This work is aimed at modelling the fatigue usage factor of $\gamma$-TiAl alloy fabricated through LAM. The modelling and simulation were performed using the COMSOL Multiphysics 5.4 software by developing a $\gamma$-TiAl alloy microstructure. This was modelled to generate the material properties (density, heat capacity at constant pressure and thermal conductivity) from the microstructure of a unit cell as a general representation of the alloy. The computed properties were used in modelling the LAM process to fabricate $\gamma$-TiAl alloy part with subsequent fatigue simulation to determine the usage factor $\left(\mathrm{K}_{\mathrm{e}}\right)$. From the models, the maximum Von Mises stress and transient temperature were $2.88 \times 10^{8} \mathrm{Nm}^{-2}$ and $1510 \mathrm{~K}$ respectively, for the LAM fabrication process; while the fatigue usage factor model showed a maximum Von Mises stress of $2.91 \times 10^{8} \mathrm{Nm}^{-2}$ and a fatigue usage factor of 0.35 .
\end{abstract}

Keywords: Additive Manufacturing; Modelling and Simulation; Computational Materials Science and Engineering; Gamma-Titanium Aluminides ( $\gamma$-TiAl); COMSOL Multiphysics

\section{Introduction}

Intermetallic titanium aluminide-based (TiAl-based) alloys have fascinated researchers working in the automotive and aerospace industries owing to their properties such as excellent oxidation resistance, good elastic modulus, high strength, good creep resistance and most importantly low density (Raji et al. 2019, 2020, 2021; Verma et al. 2017; Wang et al. 2020). Nevertheless, TiAl alloys' ductility at ambient temperature is very poor, thereby limiting its production through wrought procedures like forging; while anisotropic mechanical properties are obtained for cast TiAl-based alloys due to coarse microstructures and unpredictable defects (Ismael and Wang, 2019; Raji et al. 2020).

Furthermore, the plasticity of TiAl alloy at room temperature (RT) makes it rather problematic to adopt traditional processing techniques; thus, limiting their wider applications (Liu et al. 2020a; Raji et al. 2019, 2020). In recent years, laser additive manufacturing (LAM) processes have been developed as novel techniques for manufacturing specialized components through the use of a laser beam and it is a type of additive manufacturing (AM) technique (Raji et al. 2019; Wang et al. 2020). This makes it feasible to produce parts that have complex geometries and materials that are difficult-to-process using conventional methods (Raji et al. 2021; Wang et al. 2020). In comparison to traditional production methods, LAM reduces material wastage and cuts production time (Raji et al. 2021; Verma et al. 2017). It is understood that the phases present within the microstructures and their quantity influences the alloy mechanical properties (Holec et al. 2019; Raji et al. 2021; Wang et al. 2020).

The dual-phased $\left(\gamma\right.$ and $\alpha_{2}$ ) TiAl lamellar intermetallic alloy's strength and deformability is highly dependent on the interfaces of the lamellar ( $\mathrm{Li}$ et al. 2019). The intermetallic $\gamma$-TiAl having microstructures of lamellar primarily comprises of the $\alpha_{2}-\mathrm{Ti}_{3} \mathrm{Al}$ and $\gamma$-TiAl phases shows better ductility than any of the single-phase system (Appel and Wagner, 1998; Clemens and Mayer, 2013; Marketz et al. 2003; Li et al. 2019; Raji et al. 2020). The mechanisms of tensile fracture in TiAl alloys at high-temperatures and RT were reported to be comparable (Chen et al. 2020; Liu et al. 2020b; Wang et al. 2020); while the mechanical properties of AMed TiAlbased alloy parts exhibit inhomogeneous microstructures that is not desirable. Also, most works that were carried out to investigate the mechanical properties of TiAl alloys fabricated by AM do not determine the fatigue usage factor which is yet to be thoroughly understood. Moreover, TiAl alloys are applied in automobiles and aerospace jet engines where they are subjected to severe conditions of dynamic and cyclic loadings. Thus, examination of the fatigue usage factor of LAMed TiAl alloys are of extreme significance in a real-world standpoint.

Computer modelling can be very helpful to preliminarily determine the usage factor to fatigue of a typical dual-phase $\gamma$-TiAl intermetallic alloy. This study is a preliminary contribution 

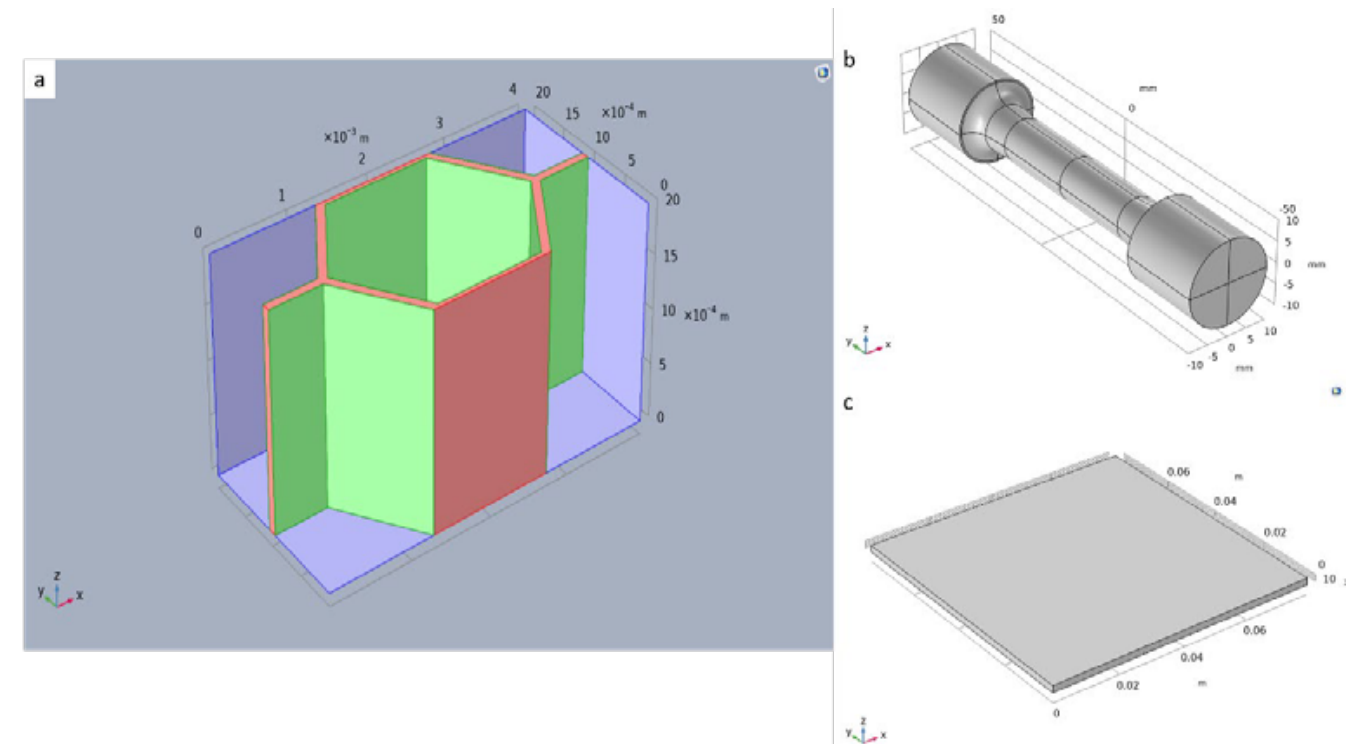

Figure 1: Geometries of the (a) Hexagonal Unit Cell Depicting the $\gamma$-TiAl Alloy Microstructure, (b) Profile of Investigated Test Piece and (c) Substrate for LAM Process

specifically devoted to the development of a suitable computational model. The work presents a three-dimensional (3D) model for TiAl alloy under a uniaxial symmetric mechanical loading. The purpose is not the simulation of the real time dynamics of the fatigue process, rather the prediction of the deformation and of the bearing capacity of TiAl alloy usage factor. As the density, heat capacity and thermal conductivity were generated from modelling of the dual-phased microstructure, the LAMed TiAl alloy maximum stress can be directly related to the mechanical properties of the real component. The properties were adopted to predict the usage factor of a real component produced with TiAl alloy. Hence, in this work, COMSOL Multiphysics 5.4 software was used for the simulation of fatigue usage factor along one direction for a dualphase TiAl intermetallic system initially modelled to evaluate its density, heat capacity and thermal conductivity with the stress determined from the LAM process.

\section{The Physical Model}

In assessing the fatigue usage factor of a dual-phase LAMed $\gamma$-TiAl alloy through modelling and simulation. There are three basic factors taken into account: (1) material properties, (2) geometry and (3) stress limits. In this study, a hexagonal shaped representation of the microstructure was modelled to depict the $\alpha_{2}-\mathrm{Ti}_{3} \mathrm{Al}+\gamma$-TiAl presented in Figure 1a. The properties (density, heat capacity and thermal conductivity) were computed from the geometrical configuration and the material property model for the unit cell with $\gamma$-TiAl representing the green areas and the $\alpha_{2}$-phase being the pink sections.

The LAM process was performed with substrate of $80 \mathrm{~mm}$ by 80 $\mathrm{mm}$ by $10 \mathrm{~mm}$ plate. The energy density of the laser beam during the LAM deposition of $\gamma$-TiAl alloy was computed based on equation (1):

$$
E=\frac{P}{V d}
$$

where, $\mathrm{E}$ is energy density of the laser beam in $\mathrm{J} / \mathrm{mm}^{2}$, $\mathrm{P}$ is power of the laser beam in Watts, $\mathrm{d}$ is the laser beam diameter in $\mathrm{mm}$ and $\mathrm{V}$ is scanning speed of laser in $\mathrm{mm} / \mathrm{s}$. This LAM process is a moving heat source which is has a Gaussian distribution profile presented in equation (2).

$f=\frac{2 * \text { Power }}{\pi r^{2}} e^{\left(-2 \frac{x^{2}+y^{2}}{r^{2}}\right)}$

where, $f$ is the heat flux in $J / \mathrm{mm}^{2}, r$ is the laser spot size radius in $\mathrm{mm}$, Power is the laser power in Watts, while $\mathrm{y}$ and $\mathrm{x}$ are variables indicating the distance of the laser beam to the centre of the build. The heat flux is applied based on equation (2) with the laser beam completing a pass (or layer) moving with velocity of $0.005 \mathrm{~m} / \mathrm{s}$. The heat source model is useful in assessing the transient temperature fields of laser material processing employing the FEA method. For simplicity in the determination of the fatigue usage factor of $\gamma$-TiAl alloy produced by LAM, uniaxial loading was applied on the test piece during investigation as shown in Figure 1b. The loading process is modelled by invoking a dynamic elasto-plastic analysis as a function of a variable displacement parameter. A sequence of successive steady state configurations can be computed assuming constant boundary/point contact conditions and non-linear material properties under a displacement control mode. A rigid constraint is placed at both edges since the material is assumed to deform elastoplastically.

\section{Governing Equations}

The effective stress in LAMed $\gamma$-TiAl alloy during deposition was predicted using solid mechanics based on equation (3). This was used to determine the behaviour of the material as a result of heat accumulation during fabrication.

$$
\rho \frac{\partial^{2} u}{\partial t^{2}}=\nabla \cdot S+F_{v}
$$

where, $\rho$ is the density in $\mathrm{kg} / \mathrm{m}^{3}$, $\mathrm{u}$ is a vector quantity, $\mathrm{S}$ is the translational transformer, $\mathrm{t}$ is time and $\mathrm{F}$ is the linear transformer. 
The heat source of the LAMed $\gamma$-TiAl alloy is expressed by the Fourier heat differential equation (equation 4 and equation 5).

$$
\begin{gathered}
\rho C_{p} \frac{d T}{d t}+\rho C_{p} u \cdot \nabla T+\nabla \cdot q=Q+Q_{t e d} \\
q=-k \nabla T
\end{gathered}
$$

where, $\mathrm{Cp}$ is the specific heat in $\mathrm{J} / \mathrm{Kg} / \mathrm{K}, \rho$ is the density in $\mathrm{kg} / \mathrm{m}^{3}$, $\mathrm{T}$ is the temperature in Kelvin $(\mathrm{K}), \mathrm{t}$ is time in seconds $(\mathrm{s}), \mathrm{Q}$ is the internal heat source intensity in $\mathrm{J} / \mathrm{mm}^{2}$ and $\mathrm{k}$ is the coefficient of thermal conductivity in $\mathrm{W} /(\mathrm{m} . \mathrm{K})$; while the $\mathrm{Cp}$ and $\mathrm{k}$ changes with temperatures. In the fatigue usage factor model, the stress state on the surface in the central part of the specimen can be evaluated using analytical expressions. Because there are no tractions on the boundaries the specimen would be in a state of plane stress. Therefore, the search for the critical plane can be reduced to two dimensions. In that case, the normal and shear stresses on a plane can be obtained as stated in equation (6) and equation (7), respectively.

$$
\begin{aligned}
& \sigma(\varphi)=\sigma_{a} \cos \varphi^{2}+\sigma_{b} \sin \varphi^{2}+\tau_{a b} \sin \varphi \cos \varphi \\
& \tau(\varphi)=\frac{\sigma_{b}-\sigma_{a}}{2} \sin 2 \varphi+\tau_{a b} \cos 2 \varphi
\end{aligned}
$$

where, $\sigma_{\mathrm{a}}$ and $\sigma_{\mathrm{b}}$ are orthogonal normal stresses and $\tau_{\mathrm{ab}}$ is the shear stress. The orientation angle $\varphi$ is calculated from the axis of $\sigma_{a}$ and $\sigma_{\mathrm{b}}$. The $\sigma_{\mathrm{a}}$ is the axial stress and the $\tau_{\mathrm{ab}}$ is shear stress via the twisting moments. The criteria for the normal stresses reflect on the plane having the range of the largest normal stress. In the determination of fatigue usage factor, a critical plane where the normal stress is highest combined with the highest range of shear stress is adopted. According to Lang (2001), usage factor determination within the elastic-plastic strain range is determined by the ke factor as expressed in equation (8). Based on these understanding we modified the equation (8) to derive equation (9).

$$
K_{e}=\frac{\Delta \varepsilon^{e l . p l .}}{\Delta \varepsilon^{f i c t . e l}}
$$

$$
\text { Fatigue usage factor }=\frac{\text { Critical Shear Stress }}{\text { Normal stress }}
$$

where, $\Delta \varepsilon^{\text {el.pl. }}$ is the elastic plastic strain range and $\Delta \varepsilon^{\text {fict.pl. is the }}$ fictitious elastic strain range, decisively influences the usage factor.

\section{Model Implementation and Solution Strategy}

The simulated material properties from microstructure gave the thermo-physical properties of thermal conductivity, heat capacity at constant pressure and density of the dual-phase TiAl alloy. The 3D fatigue usage factor model of $\gamma$-TiAl was solved using the structural mechanics module of COMSOL Multiphysics 5.4. The static elastoplastic analysis and the parametric solver were selected. This kind of solution method is referred to as pseudo-transient solution of a user defined displacement parameter and works as a pseudo-time dependent parameter. The final solution was searched iteratively by a multistage sequence of static elastoplastic solutions, under the control of the varying displacement parameters.
The materials properties for the determination of the fatigue usage factor were taken from the results generated by the material properties model based on the microstructure. The material properties were assumed to be isotropic. The direct (PARDISO) solver was used to solve the linear system. The "large deformation" box was enabled to allow large displacement solutions. At each stage, the geometric configuration in the stages were calculated by using that of the previous stage. The damped Newton method was used to solve each stage. The stress at the test piece interfaces was solved by the augmented Lagrangian method which includes the augmentation components and the segregated solver.

\subsection{Boundary Conditions}

In the LAM process model, convection and radiation are the major causes of heat loss which were considered during this simulation study of heat transfer (Balichakra et al. 2019; Morville et al. 2012). Nevertheless, as shown in Figure 2, the boundary 1, 2, 3, 5 and 6 were considered to be thermally insulated while boundary 4 was the heat flux surface. The simulation of the LAM process requires the boundary conditions to consider the laser heat source, convection and radiation losses (Morville et al. 2012); which has been accounted for in equations in equation (3). The fixed constraint implemented for the fatigue specimen as boundary condition is depicted in Figure 3.

The boundary conditions imposed for the determination of the fatigue usage factor are as follows:

- The application of stress results only in axial or longitudinal displacements of the test piece. Thus, the cylindrical bar does not rotate during testing.

- The radial displacement is fixed to zero.

- Fixed constraints at both ends of the cylindrical bar (shown in Figure 3) but all other boundaries are considered free.

- The stiffness of the specimen has to be lower than the applied force.

\subsection{Meshing and Model Geometry}

In the material properties model from the microstructure, extremely fine mesh size was adopted (as shown in Figure 4a) with a free tetrahedral shape. The maximum and minimum elements sizes were $8 \times 10^{-5} \mu \mathrm{m}$ and $8 \times 10^{-7} \mu \mathrm{m}$, respectively, with a 1.3 element growth rate and curvature factor of 0.2 . Whereas, in the model finite element mesh for the LAM process, the curvature factor was 0.4 with minimum and maximum element size of $0.00032 \mu \mathrm{m}$ and $0.0044 \mu \mathrm{m}$, respectively, also with free tetragonal mesh shape. The COMSOL Multiphysics ${ }^{\circledR}$ applies the ALE method for the moving mesh with a hyper-elastic smoothing method.

The 3D model was used to determine the fatigue usage factor based on stress-strain analysis taking into account the whole geometry (see figure $4 \mathrm{~b}$ ). This model also uses the normal meshing size of the free tetragonal element having 0.6 curvature factor, maximum growth rate of 1.5 with a minimum and maximum element size of $1.8 \mu \mathrm{m}$ and $10 \mu \mathrm{m}$, respectively. The absolute tolerance was $10^{-3}$ and the relative tolerance was $10^{-2}$. All the simulations were performed on four cores of a 16 GB RAM computer with windows 10 operating system taking about 2 hours to complete the simulation study. 
a

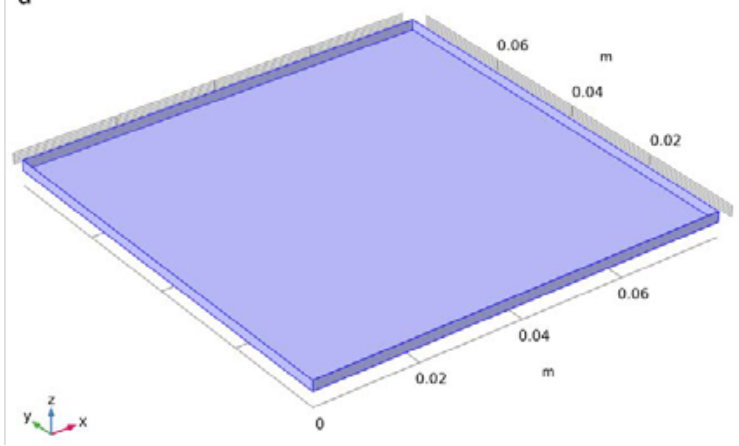

b

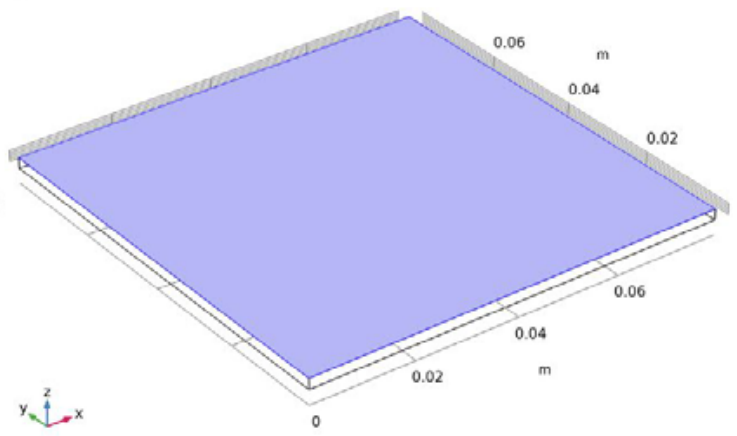

Figure 2: Boundaries of Thermally (a) Insulated Parts (b) Heat Flux Region

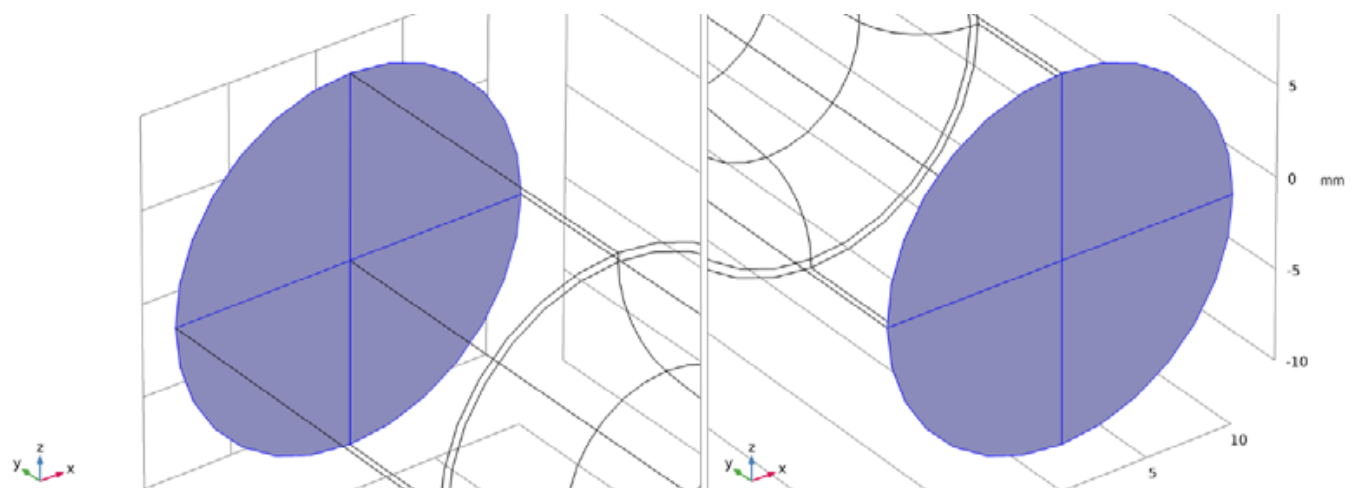

Figure 3: Fixed Rigid Connector as Constraints on the Cylindrical Bar
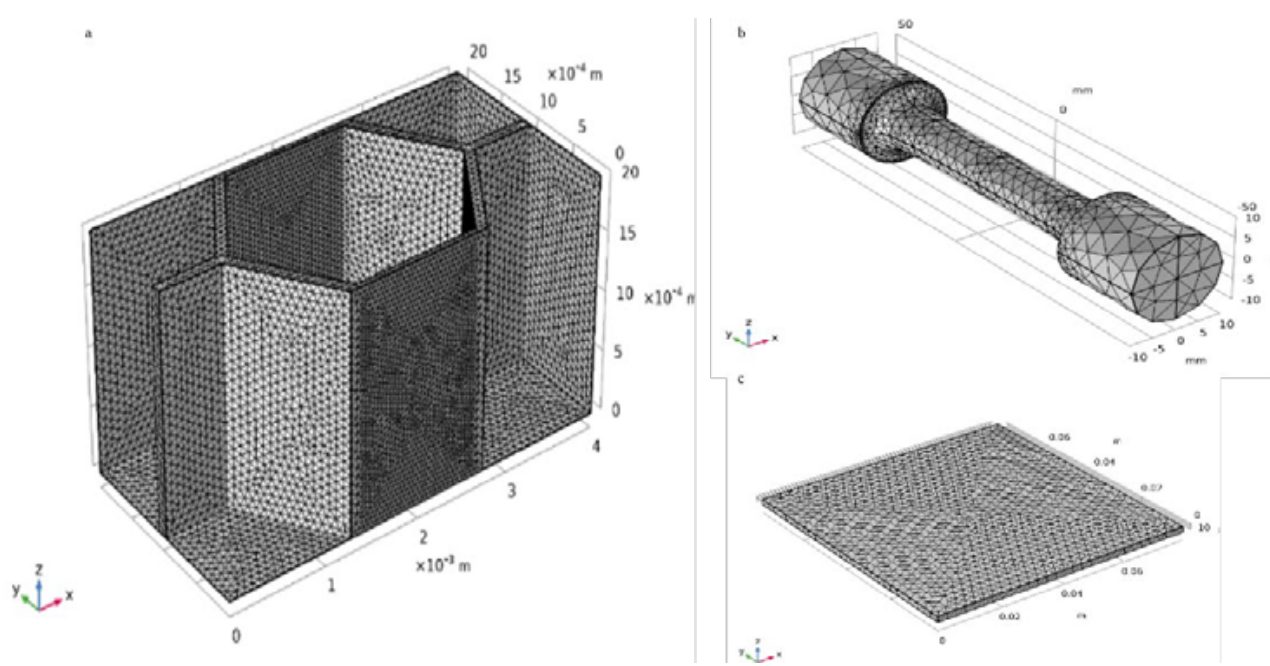

Figure 4: 3D Model Geometries Showing the Free Tetrahedral Mesh for the (a) Hexagonal Unit Cell of the Microstructure, (b) Substrate for the LAM Process, and (c) Fatigue Specimen

\subsection{Material Properties}

In the LAM process, it is essential to know the material thermophysical properties. Hence, COMSOL Multiphysics software was used to model the density, thermal conductivity and heat capacity and these results were then imported for the LAM process. But the remaining material properties of $\gamma$-TiAl were those used by Balichakra et al. (2019). The thermo-physical properties at RT used for the LAM model are listed in Table 1.

These properties have tremendous effects on the response of $\gamma$-TiAl alloy to the applied force. A normal force of $16000 \mathrm{~N}$ was applied for the simulated fatigue test at a twisting moment of $25 \mathrm{Nm}$ with limit normal stress set at $1200 \mathrm{MPa}$. This done to make sure the elastic limit of the alloy was not exceeded. Thus, the testing was carried out within the elastic-plastic regime.

\subsection{Model Assumptions}

In modelling and simulations, assumptions are made to reduce complexities of the entire process. The assumptions made to keep model simple and close to practical scenerios as possible are listed below: 
Table 1: Thermophysical Properties of $\gamma$-TiAl Alloy at Room Temperature

\begin{tabular}{|c|c|}
\hline Description & Values \\
\hline $\begin{array}{l}\text { Coefficient of Thermal } \\
\text { Expansion }\end{array}$ & $\begin{array}{l}1.1 \times 10^{-5} \mathrm{~K}^{-1} \text { (Balichakra et al. } \\
2019)\end{array}$ \\
\hline Density of Material & $3906 \mathrm{kgm}^{-3}$ \\
\hline Heat Capacity of Material & $466.6 \mathrm{~J} / \mathrm{Kg} . \mathrm{K}$ \\
\hline $\begin{array}{l}\text { Thermal Conductivity } \\
\text { Material }\end{array}$ & $15 \mathrm{~W} / \mathrm{m} . \mathrm{K}$ \\
\hline Young's Modulus & $\begin{array}{l}1.72 \times 10^{11} \mathrm{~Pa} \text { (Balichakra et al } \\
2019)\end{array}$ \\
\hline Poisson Ratio & 0.22 (Balichakra et al. 2019) \\
\hline
\end{tabular}

1. The material is isotropic

2. No heat loss by conduction during LAM process

3. Material vaporization was neglected for LAM modelling

4. The material is linearly elastic

5. For LAM modelling boundary 4 is the only heat flux region

6. LAM process flow function during melting and solidification is negligible

\section{Results and discussion}

This section presents the model results along with discussion for the properties from microstructure, LAM process and the fatigue usage factor. The thermal conductivity, heat capacity at constant pressure and density from simulation of the properties based on the microstructure are presented in Table 2. After the material properties' simulation, the heat capacities differ considerably from the values available in literature. However, the density and thermal conductivity were relatively close to values in open literature. The results of modelled material properties at room temperature are presented in Table 2 and compared with the range of values found in literature. Since density and thermal conductivity are the most important thermo-physical properties of any material. These results were accepted for the simulation of the LAM process because the derived density value conforms to the density of the $\gamma$-phase (major phase in a typical $\gamma$-TiAl with up to $90 \%$ of the alloy) and the thermal conductivity value is also acceptable.

Figure 5 shows the LAM process temperature profile during laser heating (Figure 5a), the moving laser heat source (Figure 5b), the stress build-up owing to temperature from laser heating (Figure $5 \mathrm{c}$ ), and the orthogonal view of the LAM $\gamma$-TiAl build simulated at ambient temperature (Figure 5d). it was observed that the temperature increases from RT to about $1100 \mathrm{~K}$ with subsequent rapid cooling to about $600 \mathrm{~K}$ as the laser beam completes the first layer which evident from the curve in Figure 5a. The maximum temperature further increases with the addition of the second and third layers up to about $1300 \mathrm{~K}$ and $1400 \mathrm{~K}$, respectively. This indicates that the peak temperatures of preceding layers continually increase with the addition of more layers.

The path showing a rise in temperature (Figure 5a) with time conforms with the deposition period of each layer. While the path indicative of temperature reduction with time conforms with the idle time between the successive deposition of layers which adds up to 40 seconds to complete three layers. Thermal cycles and peak temperatures underwent by the respective layer of deposit affects the resultant dimensional accuracy and mechanical properties of the component (Verma et al. 2017).

Figure 6 shows the plot of the normal stress, shear stress and Von Mises stress (Figure 6a), Von Mises stress $\left(\mathrm{N} / \mathrm{m}^{2}\right)$ in the isometric view (Figure $6 \mathrm{~b}$ ), and Von Mises stress $\left(\mathrm{N} / \mathrm{m}^{2}\right)$ in the orthographic view (Figure 6c) during the simulated fatigue testing. Modelling and simulations are done on materials as part of computational materials science to depict the actual components without having to spend resources on actual experimentations. In this study, dualphase $\gamma$-TiAl are being subjected to a uniaxial directional loading condition, at ambient temperature to predict the fatigue usage factor of the bulk material. The results of this study would a basis for several other studies on the $\gamma$-TiAl. Also, the results may be employed for further in-depth investigations.

The thermal history and high-energy input in AM results in complex phase transformations in TiAl-based alloys (Wang et al. 2020). In the work of Chen et al. (2020), molecular dynamic (MD) simulation was adopted to examine the influence of temperature on the mechanical properties, deformation mechanism and atomic diffusivity of $\mathrm{TiAl} / \mathrm{Ti}_{3} \mathrm{Al}$ boundary on a nanoscale. It was observed that the temperature has significant effects on the diffusion behaviour of both the $\alpha_{2}-\mathrm{Ti}_{3} \mathrm{Al}$ and $\gamma$-TiAl phases while undergoing uniaxial tension. Likewise, during the LAM process a Von Misses stress of $2.88 \times 10^{8} \mathrm{~N} / \mathrm{M}^{2}$ was observed with a corresponding maximum temperature of $1510 \mathrm{~K}$. The temperature and stress tend to exhibit direct proportional relationship. Interfacial strain was produced through the boundary grains twisting preceded by competitive growth resulting from compression of adjacent grains. Consequently, atoms of the grains adjacent to each other are expected to move along the direction of the applied force, to facilitate the movement of interface towards the axial direction (Liu et al. 2020b). Typical LAMed TiAl alloys displays a band of alternative microstructure containing of hexagonal colony grains of the $\alpha_{2} / \gamma$ laths phases (Zhang et al. 2020).

Figure 7 shows the modelled test piece for the prediction of the LAMed TiAl fatigue usage factor. The result shows that the stressstrain relation is isotropic. According to Li et al. (2019), temperature does not affect the mechanical properties of flow stress, yield strain, yield strength, and elastic modulus. However, the overriding failure mode is plastic deformation and fracture under constrained

Table 2: Comparison of the Modelled Material Properties of $\gamma$-TiAl Alloy with Available Data

\begin{tabular}{|c|c|c|}
\hline Description & Model Values & Range of values in Literature \\
\hline Density $\left(\mathrm{Kg} / \mathrm{m}^{3}\right)$ & 3609 & $3600-4200$ \\
\hline Heat Capacity (J/Kg.K) & 466.6 & $610-700$ \\
\hline Thermal Conductivity (W/m.K) & 15 & $10-20$ \\
\hline
\end{tabular}



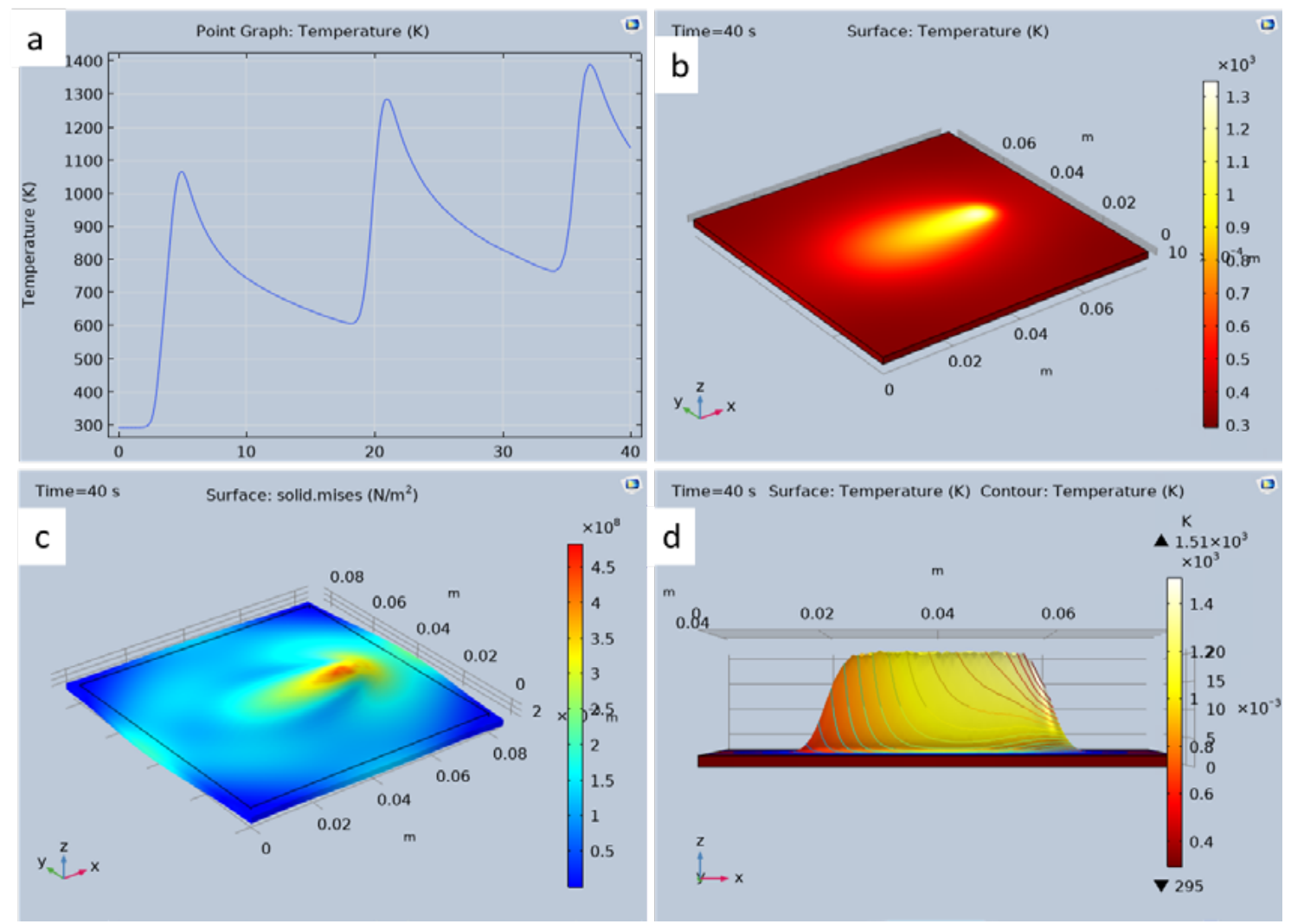

Figure 5: Showing the LAM process (a) Temperature Profile during Deposition, (b) Moving Heat Source, (c) Stress Accumulation due to Laser Heating, and (d) Orthogonal View of LAM Built Part

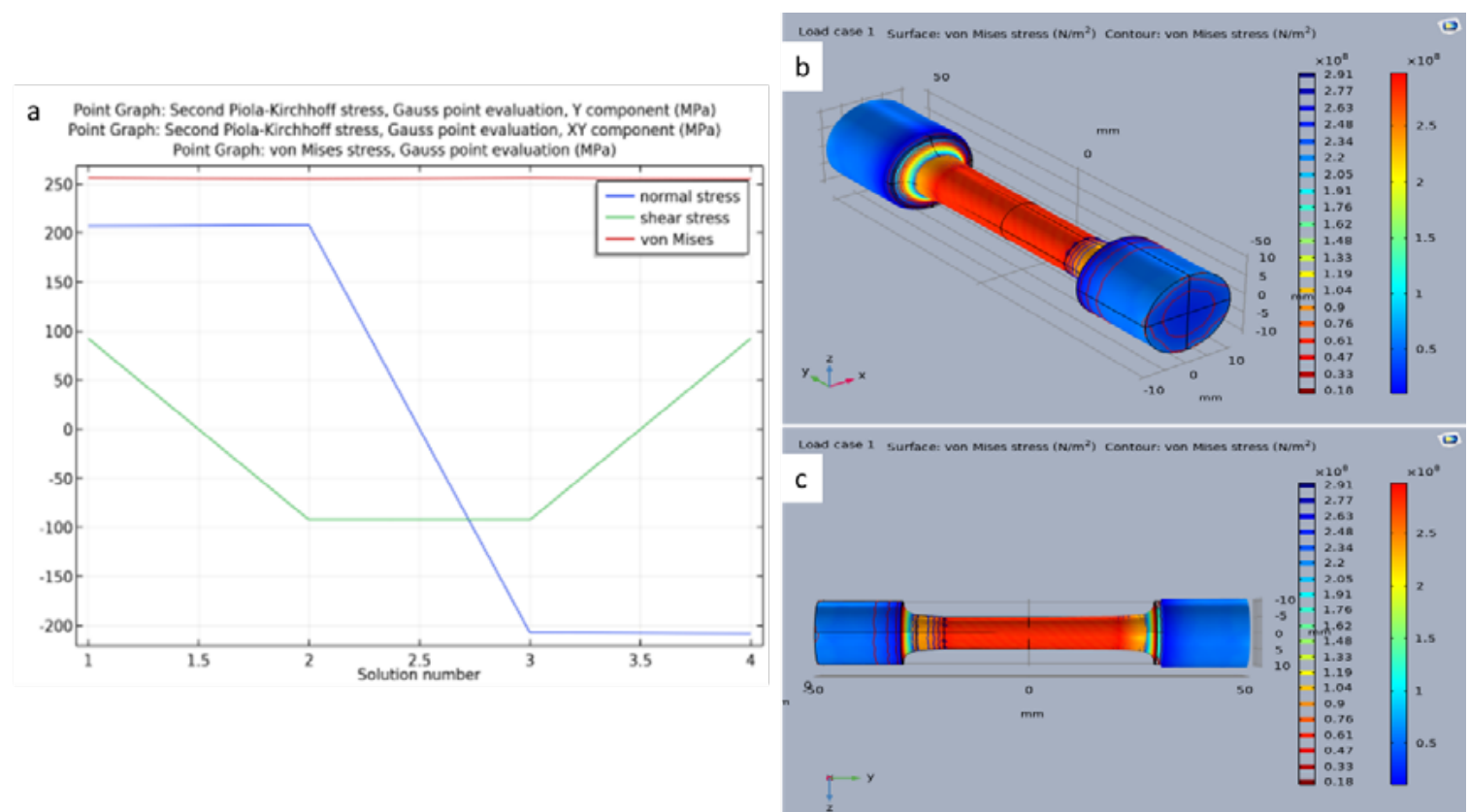

Figure 6: Showing the fatigue test piece (a) Point graph for the normal stress, shear stress and Von Mises stress (MPa), (b) Von Mises stress $\left(\mathrm{N} / \mathrm{m}^{2}\right)$ in the isometric view, and (c) Von Mises stress $(\mathrm{N} / \mathrm{m} 2)$ in the orthographic view

boundary conditions (Chen et al. 2020; Li et al. 2019). Since the applied loading does not exceed the material elastic limit by using a normal force of $16000 \mathrm{~N}$ at a twisting moment of $25 \mathrm{Nm}$ with limit normal stress set at $1200 \mathrm{MPa}$. The predicted values for the fatigue usage factor was 0.35 with corresponding peak Von Mises stress of $2.91 \times 10^{8} \mathrm{~N} / \mathrm{M}^{2}$ was produced.
From literature, it is understood that the gamma-phased $\gamma$-TiAl lamellar system fails due to plastic deformation whereas fracture is the mode of failure in $\mathrm{Ti}_{3} \mathrm{Al}$ phase system (Chen et al. 2020; $\mathrm{Li}$ et al. 2019). This is due to the ductile nature of the $\gamma$-phase as compared to the brittle $\alpha_{2}$-phase. The model was executed through various simulations using COMSOL Multiphysics and the resulting deformations are observed. It was noticed in the that modelling both phases of TiAl, and the differences in behaviour between both 


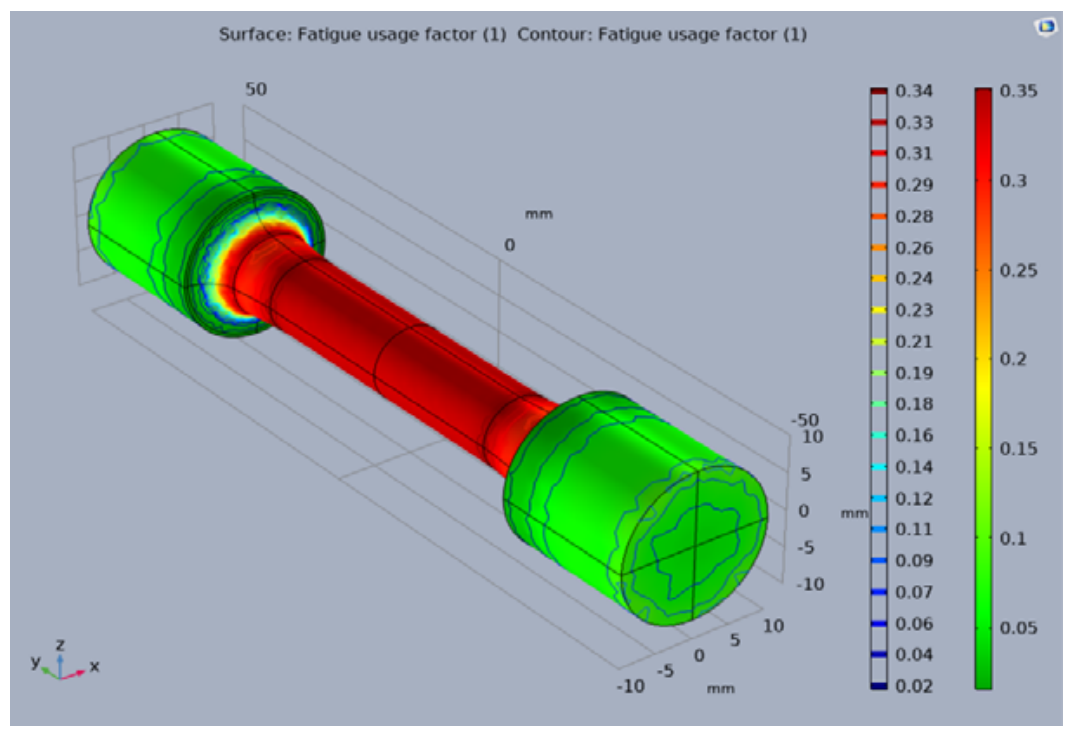

Figure 7: Modelled Fatigue Test Piece Showing the Fatigue Usage Factor

$\alpha_{2}-\mathrm{Ti}_{3} \mathrm{Al}$ and $\gamma$-TiAl phases demonstrated similar characteristics owing to their intermetallic nature. Thus, it was established that the behaviour of the dual-phased $\gamma$-TiAl interface system fabricated using LAM gives a fatigue usage factor of 0.35 . This relationship is expected to be in agreement with the experiment results based on this theoretical analysis.

\section{Conclusions}

In this paper, fatigue usage factor of LAMed two-phase $\gamma$-TiAl alloy under uniaxial loading was simulated with COMSOL Multiphysics version 5.4. The material properties mainly thermal conductivity, heat capacity at constant pressure and density were computed by the simulation of the $\gamma$-TiAl alloy microstructure. These properties were adopted in the investigation and determination of fatigue usage factor simulation of the LAMed $\gamma$-TiAl alloy. Based on the simulation results, it was concluded that the thermal properties were not the same as values in several literatures. This is because our simulated heat capacity value was about $50 \%$ lesser than most values reported for $\gamma$-TiAl-based alloys' heat capacity in literature. This disparity was attributed to assumptions made during the material property modelling. However, the density and thermal conductivity were in agreement with known values of TiAl-based alloys available. In the LAM process, the peak temperatures of preceding layers tend to consistently increase with addition of more layers. This shows a maximum transient temperature $1510 \mathrm{~K}$ with corresponding peak Von Mises stress $2.88 \times 10^{8} \mathrm{~N} / \mathrm{M}^{2}$. However, a value of 0.35 was measured as the fatigue usage factor with a peak Von Mises stress of $2.91 \times 10^{8} \mathrm{~N} / \mathrm{M}^{2}$ was noticed. Finally, the results of this study validate the conclusions drawn in several other studies for dual-phase $\gamma$-TiAl, and the results may be used for further indepth investigations.

\section{Acknowledgments}

The authors would like to acknowledge the financial support (scholarship grant) from the African Laser Centre-National Laser Centre; Council of Scientific and Industrial Research (ALC-NLC; CSIR), Project Number LHIP500 Task ALC S100.

\section{References}

1. Appel, F. and Wagner, R, 1998. Microstructure and deformation of two-phase $\gamma$-titanium aluminides. Materials science and engineering: $R$ : reports, 22, 187-268. https://doi.org/10.1016/ S0927-796X(97)00018-1

2. Balichakra, M., Bontha, S., Krishna, P. and Balla. V.K. 2019. Laser surface melting of $\gamma$-TiAl alloy: an experimental and numerical modeling study. Materials Research Express, 6, 046543. https://doi. org/10.1088/2053-1591/aafc89

3. Chen, J., Chen, W. and Wang, C. 2020. Modeling and investigation for atomic diffusion and mechanical properties of TiAl/Ti3Al interface: temperature effect. Applied Physics A, 126, 1-9. https://doi. org/10.1007/s00339-020-03678-0

4. Clemens, H. and Mayer, S. 2013. Design, processing, microstructure, properties, and applications of advanced intermetallic TiAl alloys. Advanced Engineering Materials, 15, 191-215. https://doi. org/10.1002/adem.201200231

5. Holec, D., Abdoshahi, N., Mayer, S. and Clemens, H. 2019. Thermal expansion and other thermodynamic properties of $\alpha 2$-Ti3Al and $\gamma$-TiAl intermetallic phases from first principles methods. Materials, 12, 1292. https://doi.org/10.3390/ma12081292

6. Ismaeel, A. and Wang, C.S. 2019. Effect of $\mathrm{Nb}$ additions on microstructure and properties of $\gamma$-TiAl based alloys fabricated by selective laser melting. Transactions of Nonferrous Metals Society of China, 29, 1007-1016. https://doi.org/10.1016/ S1003-6326(19)65009-0

7. Lang, H. 2001. Fatigue- determination of more realistic usage factor. Nuclear Engineering and Design, 206, 221-234. https://doi. org/10.1016/S0029-5493(00)00436-2

8. Li, W., Yin, Y., Xu, Q., Zhou, J., Nan, H., Ji, X., Shen, X., Feng, X. Yu, W., Tu, Z. and Pang, N. 2019. Tensile behavior of $\gamma / \alpha 2$ interface system in lamellar TiAl alloy via molecular dynamics. Computational Materials Science, 159, 397-402. https://doi.org/10.1016/j. commatsci.2018.12.043

9. Liu, Z.Q., Xu, G.J., Wang, W.B. and Su, Y.H. 2020a. Effects of annealing on microstructure and mechanical properties of $\gamma$-TiAl alloy fabricated via laser melting deposition. Transactions of Nonferrous Metals Society of China, 30, 917-927. https://doi.org/10.1016/ S1003-6326(20)65265-7

10. Liu, Y., Xue, X., Fang, H., Tan, Y., Chen, R., Su, Y. and Guo, J. $2020 \mathrm{~b}$. The growth behavior of columnar grains in a TiAl alloy during directional induction heat treatments. CrystEngComm, 22, 1188-1196. https://doi.org/10.1039/C9CE01631G

11. Marketz, W.T., Fischer, F.D. and Clemens, H. 2003. Deformation mechanisms in TiAl intermetallics - experiments and modeling. International Journal of Plasticity, 19, 281-321. https://doi. org/10.1016/S0749-6419(01)00036-5

12. Morville, S., Carin, M., Peyre, P., Gharbi, M., Carron, D., Le Masson, P. and Fabbro, R. 2012. 2D longitudinal modeling of heat transfer and fluid flow during multi-layered. Journal of Laser Applications, Laser Institute of America, 24, 1-9. https://doi.org/10.2351/1.4726445

13. Raji, S.A., Popoola, A.P.I., Pityana, S.L., Popoola, O.M., Aramide, F.O., Tlotleng, M. and Arthur, N.K.K., 2019. Laser based additive manufacturing technology for fabrication of titanium aluminide-based 
composites in aerospace Component Applications. In: Mofid Gorji-Bandpy, $M$ and Aly, A (Eds), Aerodynamics 2019, IntechOpen. London, pp. 193-218. https://doi.org/10.5772/intechopen.85538

14. Raji, S.A., Popoola, A.P.I., Pityana, S.L. and Popoola, O.M., 2020. Characteristic effects of alloying elements on $\beta$ solidifying titanium aluminides: A review. Heliyon, 6(7), e04463. https://doi.org/10.1016/j. heliyon.2020.e04463

15. Raji, S.A., Popoola, A.P.I., Pityana, S.L. and Tlotleng, M., 2021. Microstructure and mechanical properties of heat-treated Ti-Al-Si alloy produced via laser in situ alloying. Journal of Materials Engineering and Performance, 30(5), 3321-3332. https://doi. org/10.1007/s11665-021-05681-9
16. Verma, V., Mandal, A. and Shukla, A. 2017. Laser direct metal deposition (LDMD)-An overview. International Journal of Advance Research and Innovation, 5, 459-467. https://ijari.org/assets/ papers/5/4/IJARI-ME-17-12-151.pdf

17. Wang, J., Luo, Q., Wang, H., Wu, Y., Cheng, X. and Tang, H. 2020. Microstructure characteristics and failure mechanisms of Ti-48Al$2 \mathrm{Nb}-2 \mathrm{Cr}$ titanium aluminide intermetallic alloy fabricated by directed energy deposition technique. Additive Manufacturing, 32, 101007 https://doi.org/10.1016/j.addma.2019.101007

18. Zhang, X., Li, C., Zheng, M., Zhong, H. and Gu, J. 2020. Alternativeband microstructure and LPSO phase in TiAl alloy produced by direct laser deposition. Materials Characterization, 164, 110315. https://doi. org/10.1016/j.matchar.2020.110315 